\title{
Attentional Cueing at the Saccade Goal, Not at the Target Location, Facilitates Saccades
}

\author{
Aarlenne Z. Khan, ${ }^{1,2}$ Stephen J. Heinen, ${ }^{1}$ and Robert M. McPeek ${ }^{1,3}$ \\ ${ }^{1}$ The Smith-Kettlewell Eye Research Institute, San Francisco, California 94115, ${ }^{2}$ Centre for Neuroscience Studies, Queen's University, Kingston, Ontario K7L \\ 3N6, Canada, and ${ }^{3}$ Department of Biological Sciences, SUNY College of Optometry, New York, New York 10036
}

Presenting a behaviorally irrelevant cue shortly before a target at the same location decreases the latencies of saccades to the target, a phenomenon known as exogenous attention facilitation. It remains unclear whether exogenous attention interacts with early, sensory stages or later, motor planning stages of saccade production. To distinguish between these alternatives, we used a saccadic adaptation paradigm to dissociate the location of the visual target from the saccade goal. Three male and four female human subjects performed both control trials, in which saccades were made to one of two target eccentricities, and adaptation trials, in which the target was shifted from one location to the other during the saccade. This manipulation adapted saccades so that they eventually were directed to the shifted location. In both conditions, a behaviorally irrelevant cue was flashed $66.7 \mathrm{~ms}$ before target appearance at a randomly selected one of seven positions that included the two target locations. In control trials, saccade latencies were shortest when the cue was presented at the target location and increased with cue-target distance. In contrast, adapted saccade latencies were shortest when the cue was presented at the adapted saccade goal, and not at the visual target location. The dynamics of adapted saccades were also altered, consistent with prior adaptation studies, except when the cue was flashed at the saccade goal. Overall, the results suggest that attentional cueing facilitates saccade planning rather than visual processing of the target.

\section{Introduction}

A sudden visual event, such as a street light turning on, often draws attention. Such exogenous attention shifts are useful in detecting potentially important changes in our surroundings so that we may react appropriately to them. It has been shown that an exogenous attention shift that is elicited by the presentation of a salient visual cue reduces saccadic eye movement latencies to targets presented shortly afterward at the same location (Posner and Cohen, 1984; Bell et al., 2004; Fecteau et al., 2004). Although this classic cueing effect has been studied for over 20 years, it remains unclear whether exogenous cueing reduces saccade latencies by facilitating processing of the target, consistent with an influence on earlier visual processing stages (Jonides, 1981), or by facilitating the response to the target (i.e., the execution of the eye movement to that location), consistent with an influence on later motor processing stages (Fecteau and Munoz, 2005).

It has been difficult to answer this question, since both the visual target and the motor goal are usually presented at the same location (but see Fecteau and Munoz, 2005). One way to dissociate the locations of visual and motor processing is through saccadic adaptation (McLaughlin, 1967; Deubel et al., 1986; Straube et al., 1997; Hopp and Fuchs, 2004), where the amplitude and/or

Received Sept. 8, 2009; revised March 1, 2010; accepted March 5, 2010.

A.Z.K. was supported by the Canadian Institutes of Health Research (Canada). R.M.M. was supported by National Institutes of Health (NIH) Grant EY014885. S.J.H. was supported by NIH Grant EY117720. We are grateful to Gunnar Blohm and Edward Keller for helpful comments and Andrew Pruszynski for technical help.

Correspondence should be addressed to Robert M. McPeek, Department of Biological Sciences, SUNY College of Optometry, 33 West 42nd Street, New York, NY 10036. E-mail: rmcpeek@sunyopt.edu.

DOI:10.1523/JNEUROSCI.4437-09.2010

Copyright $\odot 2010$ the authors $\quad 0270-6474 / 10 / 305481-08 \$ 15.00 / 0$ direction of a saccade to a visual target is gradually modified by shifting the target to another location during the saccade (McLaughlin, 1967; Hopp and Fuchs, 2004). For amplitude adaptation, if the shift is $\sim 33 \%$ of the initial target distance or smaller, it is generally not perceived by human subjects (Bridgeman et al., 1975). The reduction of saccade amplitude by such adaptation trials occurs rapidly: in humans, after $\sim 40-60$ trials, saccades are adapted and the endpoints of saccades to the same initial target location differ significantly from their preadaptation values (Deubel et al., 1986; Frens and van Opstal, 1994). It has been suggested that saccadic adaptation occurs at the motor level, as a change in the saccade command that is issued for a given visual target eccentricity (Frens and van Opstal, 1994; Wallman and Fuchs, 1998) rather than a change in the perceived visual target distance (Deubel, 1991; Bahcall and Kowler, 1999, 2000). This change is presumably produced by the retinal error signal between the target location and the saccade location endpoint present during the adapting period (Wallman and Fuchs, 1998; Bahcall and Kowler, 2000; Noto and Robinson, 2001) as opposed to the amplitude of the corrective saccade (Albano and King, 1989).

To distinguish between sensory and motor hypotheses about the influence of exogenous attention on saccadic latency, amplitude and dynamics, we used saccadic adaptation to dissociate the saccade goal from the location of the visual target. We flashed a behaviorally irrelevant cue at different locations before presenting the visual target during saccadic adaptation. We hypothesized that if exogenous attention facilitates sensory processing of the target, then a cue flashed at the upcoming target location should have the greatest influence on the saccade, because it would result 
in faster detection of the target. On the other hand, if attention facilitates motor production of the saccade, then a cue flashed at the upcoming saccade goal should have the greatest influence since the saccade would be triggered faster by the cue.

We found that saccade latencies, amplitudes and dynamics were influenced the most when a cue was flashed at the upcoming saccade goal location, and not at the visual target location. This observation supports the hypothesis that exogenous attention reduces saccade latencies by facilitating motor planning.

\section{Materials and Methods}

Subjects. Seven subjects (ages: 25-40, 4 female) participated in the experiment. Five subjects were naive to the goal of the experiment. The experimental protocol was preapproved by the Smith-Kettlewell Institutional Review Board in compliance with the National Commission for the Protection of Human Subjects of Biomedical and Behavioral Research. All subjects had normal or corrected to normal vision.

Apparatus. Subjects were seated in front of a 17-inch high-resolution Nanao color monitor (1.76 min arc/pixel) with a refresh rate of $60 \mathrm{~Hz}$ that was controlled by a Macintosh (Apple) computer. Subjects were seated at a distance of $48 \mathrm{~cm}$ from the monitor in a dimly lit room. The edges of the monitor were slightly visible because of the light projected from nearby data acquisition monitors. The monitor background was black $\left(0.05 \mathrm{~cd} / \mathrm{m}^{2}\right)$. Stimuli were presented using Matlab (The MathWorks Inc.) and functions from the PsychToolbox (Brainard, 1997; Pelli, 1997). Eye position was recorded at $1000 \mathrm{~Hz}$ using a video-based tracker (Eyelink 1000; SR Research). A chinrest maintained the viewing distance and stabilized the head for accurate eye tracking. Before each block of trials, the eye tracker was calibrated by having the subject fixate a series of 9 positions on the display ( 8 surrounding the periphery of the display and the center) while the gains and offsets were set.

Procedure. Subjects performed one adapted saccade block and 2 control (normal) saccade blocks that consisted of 192-384 trials within each block. In the adapted saccade block, the target shifted during the saccade from an eccentricity of $15^{\circ}$ to $10^{\circ}$ throughout the block (33\% of the amplitude of the first target). The five naive subjects were unaware of any differences between the control and adapted blocks, verbally ascertained during debriefing. We chose to use a decreasing amplitude adaptation paradigm (Hopp and Fuchs, 2004) because, while many studies on saccadic adaptation have reliably shown a decrease or increase in saccade amplitude in one direction (Semmlow et al., 1989; Wallman and Fuchs, 1998), decreasing saccade amplitude appears to be faster, lasts longer and results in a greater amount of adaptation than increasing saccade amplitude (Semmlow et al., 1989; Erkelens and Hulleman, 1993; Fuchs et al., 1996; Panouillères et al., 2009).

The sequence of each trial in the adapting block was as follows: subjects fixated on a dot [white circle $\left(37.2 \mathrm{~cd} / \mathrm{m}^{2}\right)$, diameter $=0.25^{\circ}$ ] at $10^{\circ} \mathrm{left}$ of the center of the screen (Fig. $1 A$, trial sequence). After a variable interval of $600-800 \mathrm{~ms}(36-48$ frames, 1 frame $=16.7 \mathrm{~ms}$ ), a cue [light gray circle $\left(0.6 \mathrm{~cd} / \mathrm{m}^{2}\right)$, diameter $=0.25^{\circ}$, duration $=33.3 \mathrm{~ms}$, 2 frames] was presented randomly at 1 of 7 positions aligned horizontally with the fixation position $\left[5^{\circ}\right.$ left of fixation, at fixation (fixation dot dimmed for $33.3 \mathrm{~ms}, 2$ frames), $5^{\circ}, 10^{\circ}, 15^{\circ}, 20^{\circ}$, or $25^{\circ}$ right of fixation] (Fig. $1 \mathrm{~B}$, target and cue positions). The cue was then extinguished and was followed by a 33.3 ( 2 frame) ms delay, corresponding to a $66.7 \mathrm{~ms}$ ( 4 frame) stimulus onset asynchrony (SOA). The $66.7 \mathrm{~ms} \mathrm{SOA}$ was determined using pilot experiments to reliably show attentional facilitation for control saccades. Next, a target [white square $\left(37.2 \mathrm{~cd} / \mathrm{m}^{2}\right)$, diameter $=$ $0.25^{\circ}$ ] was presented $15^{\circ}$ to the right of fixation and the fixation dot was extinguished at the same time. Subjects were asked to saccade to the target as soon as they saw it. Eye position was monitored online and as soon as the left eye moved $1.5^{\circ}$ away from the fixation dot, the target shifted to a location $10^{\circ}$ to the right of initial fixation. The target always shifted to its second position before the end of the first saccade. After the target jump, the target remained illuminated at its second position for $500 \mathrm{~ms}$ ( 30 frames). The next trial began following a 600 (36 frames) ms interstimulus interval. In addition, there was a condition in which no cue was presented but the timing remained the same.
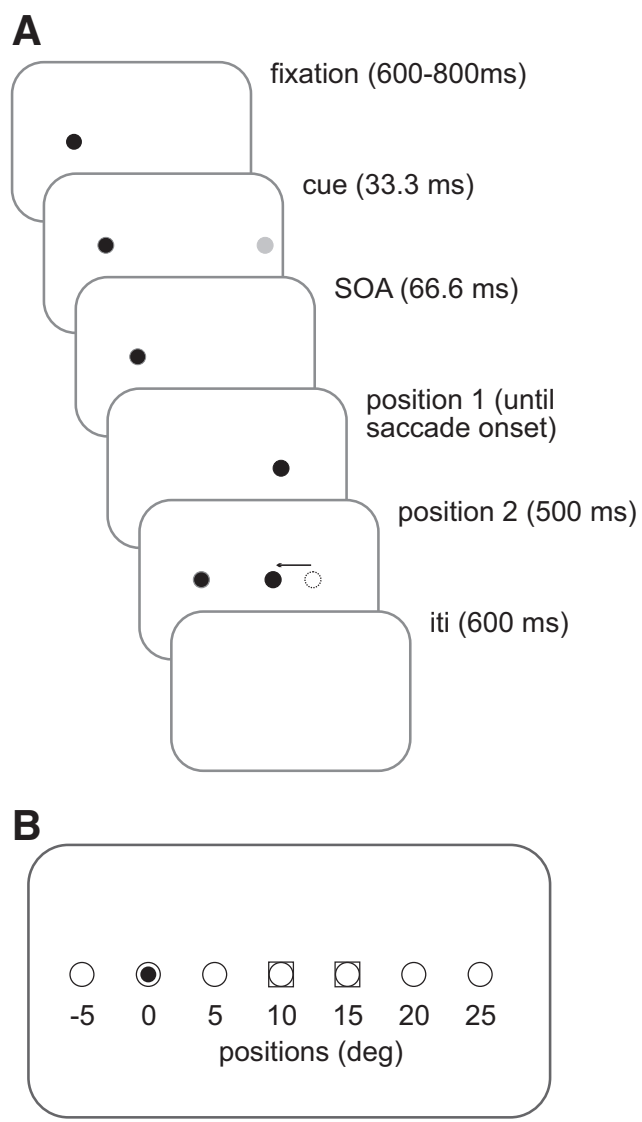

Figure 1. Task setup. $\boldsymbol{A}$, Trial sequence for an adaptation trial. Each trial began when a fixation target appeared for a random time ( $600-800 \mathrm{~ms}, 36$ - 48 frames in 6 frame intervals) (black filled circle). Next, a behaviorally irrelevant cue (light filled gray circle) was presented at one of 7 locations (see $\boldsymbol{B}$ ) for $33.3 \mathrm{~ms}$. Subjects were asked to ignore the presence of the cue. Following a SOA time of $66.67 \mathrm{~ms}$, a target was presented at $15^{\circ}$, labeled as position 1 (darker gray circle). During the saccade, the position of the target shifted $5^{\circ}$ backward (arrow) toward fixation (position 2, circle). The shortest saccade duration was $44 \mathrm{~ms}$, and the target was always visible at its new location before the end of the saccade. The target then remained illuminated for $500 \mathrm{~ms}$ ( 30 frames). During control trials, the target was not shifted, i.e., position 1 and 2 were identical, either at $10^{\circ}$ or at $15^{\circ}$. The intertrial interval (ITI) was $600 \mathrm{~ms}$ ( 36 frames). $\boldsymbol{B}$ Locations of targets and cues. The possible positions of the cues (open circles) were $5^{\circ}$ left of fixation (small black circle inside the open circle), at fixation (dimming of the fixation target), and $5^{\circ}, 10^{\circ}, 15^{\circ}, 20^{\circ}$ and $25^{\circ}$ right of fixation. Target locations are shown as gray squares surrounding the open circles. Cues could appear randomly at one of 7 positions. In addition, there was a no-cue condition, which had the same timings.

The two control saccade blocks consisted of trial sequences that were identical to the target jump sequence, except that the target did not change positions during the saccade and remained at either the $10^{\circ}$ or $15^{\circ}$ position for the remainder of the trial. Within each block, target position remained the same. The order in which the 3 blocks were presented to each subject was randomized.

Data analysis. A total of 5496 trials were collected, with a total of 2088 adapted saccade trials, 1656 of the $10^{\circ}$ control trials and 1752 of the $15^{\circ}$ control trials. The total number of trials per subject ranged from 576 to 1152. All data were analyzed using Matlab 7.3 (The Math Works Inc.). Eye velocity was obtained by digital differentiation of eye position signals and filtered to reduce noise ( 2 pole Butterworth filter, cutoff $=50 \mathrm{~Hz}$ ). Saccades were detected using a velocity threshold of $25 \%$ s. Across all subjects and groups, we removed all trials with saccade latencies of $<80$ ms (Wenban-Smith and Findlay, 1991) or $>500 \mathrm{~ms}$ (Walker et al., 1995). There were a total of 585 trials removed $(10.6 \%)$. Next, to remove outliers within each subject and each group (control, adapted), we removed all trials with saccade latencies that did not fall within 3 SDs of the mean within each subject and each group (63 additional trials). Additionally 

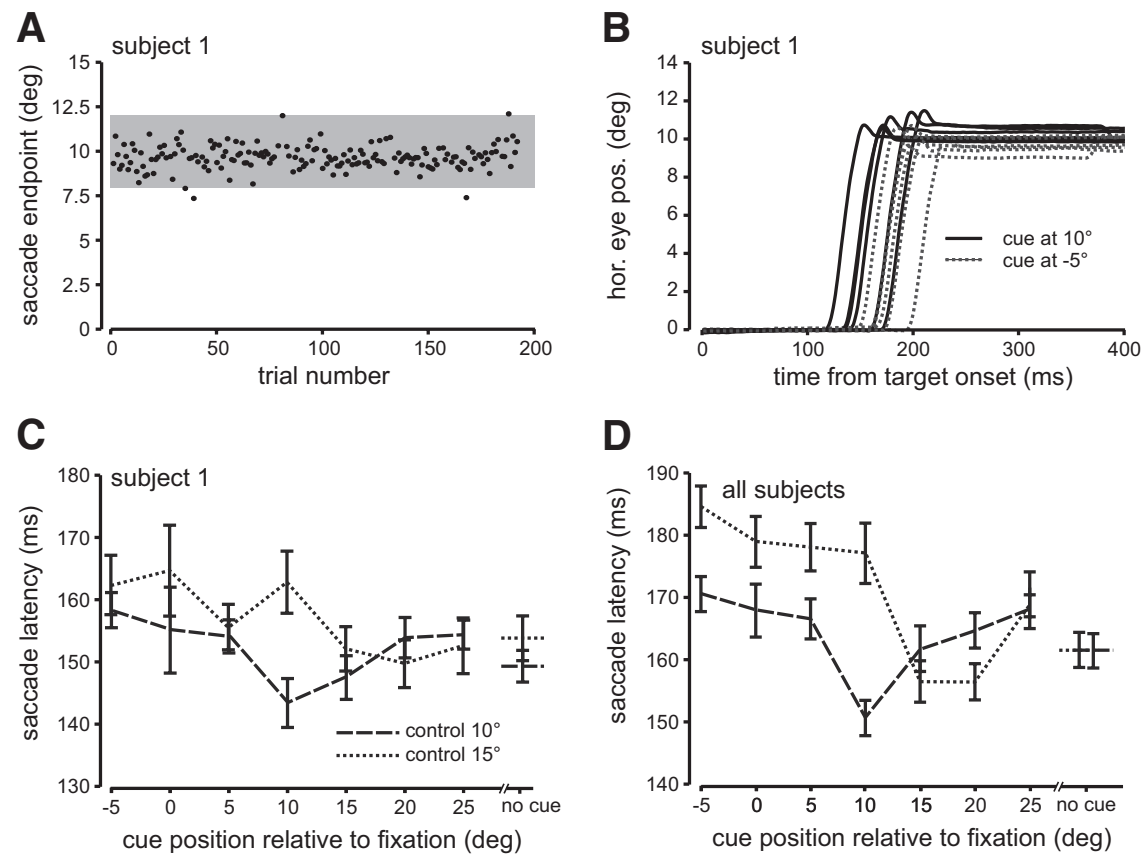

Figure 2. Control trials. $A$, Saccade amplitude is plotted as a function of trial number for the $10^{\circ}$ control saccade block from subject 1. Black dots represent saccade endpoint for each trial. The gray region demarcates the range of saccade amplitudes that were included in the analysis ( $2^{\circ} \pm$ target amplitude). $\boldsymbol{B}$, Six trials from $\boldsymbol{A}$ are shown with horizontal eye position plotted as a function of time from target onset randomly selected from two different cue conditions. The black solid traces depict trials in which the cue was flashed at $10^{\circ}$ (same location as the target) and the gray dotted traces depict trials in which the cue was flashed at $5^{\circ}$ left of fixation. C, Average saccade latencies for each flashed cue position are plotted for the two control saccade groups $10^{\circ}=$ dashed line and $15^{\circ}=$ dotted line for subject 1 as in $\boldsymbol{A}$ and $\boldsymbol{B}$. No cue in the $x$-axis represents the no-cue condition. Error bars are SEM. Please see supplemental Figure 1 (available at www.jneurosci.org as supplemental material) for data from a second subject. $\boldsymbol{D}$, Average saccade latencies across all subjects (7) are plotted in the same manner as $\mathbf{C}$.

there were a number of outliers in the dynamics data. Therefore, all peak velocities above $700 \%$ mean $(\mathrm{M})=435 \% \mathrm{~s}, \mathrm{SD}=81 \% \mathrm{~s})$, acceleration durations above $50 \mathrm{~ms}(\mathrm{M}=36 \mathrm{~ms}, \mathrm{SD}=3.17 \mathrm{~ms})$ and deceleration durations above $70 \mathrm{~ms}(\mathrm{M}=32 \mathrm{~ms}, \mathrm{SD}=10 \mathrm{~ms})$ were excluded from the data. This resulted in 4234 (77\%) remaining trials.

To classify saccades in the adaptation blocks as either adapted or unadapted, we determined a priori a specific range of target movement amplitudes. To be considered an adapted saccade, the movement endpoint was required to fall within $\pm 2^{\circ}$ of the second target position, i.e., $8^{\circ}$ to $12^{\circ}$ from fixation. This criterion yielded a total of 878 adapted saccades. We used the same or similar criteria for selecting the control saccade trials for each target position, i.e., $8^{\circ}$ to $12^{\circ}$ for the $10^{\circ}$ control target trials $(n=1241)$ and $13^{\circ}$ to $17^{\circ}$ for the $15^{\circ}$ control target trials $(n=$ 1020). We also selected from the adapted saccade trials a set of saccades with amplitudes that fell within $\pm 2^{\circ}$ of the first target position, i.e., $13^{\circ}$ to $17^{\circ}$, which we labeled as unadapted saccades (total unadapted saccades, $n=331$ ). These four groups of saccades were then used in the subsequent analyses. Note that the range of $4^{\circ}$ ensures no endpoint overlap between the adapted and unadapted saccade groups and allows a fair comparison of saccades in the control and adapted trials. The mean amplitudes for all groups were as follows: $10^{\circ}$ control $=9.8^{\circ}$, adapted trials $=10.61^{\circ}, 15^{\circ}$ control $=14.6^{\circ}$ and unadapted trials $=13.9^{\circ}$.

In addition, we compared peak velocities and saccade durations between the adapted and the $10^{\circ}$ control saccades. To do so, we compared saccades with the same amplitudes across the two groups. For each group at each cue location, we binned the amplitudes into 12 equal bins with widths of $0.25^{\circ}$. We then selected the same number of trials from each bin for both groups; this number was equivalent to the group with the fewer number of trials within that bin. The same number of trials was then randomly selected from the second group. This resulted in equivalent amplitudes $(p>0.05)$ at each cue location across both groups. Subsequent calculations of peak velocities and durations were then made from these selected trials. Acceleration duration was defined as the time from saccade onset to peak velocity, and deceleration duration was defined as the time from peak velocity to the end of the saccade.

\section{Results}

\section{Control saccade trials_-latencies}

Saccade amplitudes for a typical subject as a function of trial number for the $10^{\circ}$ control saccade block are shown in Figure $2 \mathrm{~A}$. Most saccade endpoints were close to the target at $10^{\circ}$. Note that saccade endpoint is synonymous with saccade amplitude since fixation was set to $0^{\circ}$. The highlighted gray area depicts the $\pm 2^{\circ}$ range within which lie the endpoints of saccades used in subsequent analyses, i.e., $8^{\circ}$ to $12^{\circ}$. Figure $2 B$ shows typical control saccades to the $10^{\circ}$ target when the cue was flashed $10^{\circ}$ right (at the target's position) or $5^{\circ} \mathrm{left}$ of fixation, plotted as a function of time. Six randomly chosen trials are shown for each cue position $\left(10^{\circ}\right.$ right, solid traces and $5^{\circ}$ left, dotted traces). Saccade latencies were consistently shorter when the flashed cue appeared at the same location as the subsequent target location. In Figure $2 C$, mean latencies for the same subject are plotted as a function of all cue locations and both target locations $\left(10^{\circ}\right.$ control block, dashed lines and $15^{\circ}$ control saccade, dotted line). The data from this subject show that saccade latency varied as a function of the position of the flashed cue, with the shortest latencies occurring when the cue appeared at or near the target location. When the target was presented at $10^{\circ}$, saccade latencies were shortest when the cue was presented at $10^{\circ}$. For the $15^{\circ}$ condition, saccade latencies were shortest when the cue appeared at the $15-25^{\circ}$ locations. This was the case even though the flashed cue was behaviorally irrelevant.

Figure $2 D$ summarizes both control saccade conditions across all subjects. Particularly for the $10^{\circ}$ target group, saccade latencies were shortest when the cue was flashed at the same location as the target and increased gradually in either direction as the distance between the cue and the target increased. A similar pattern can also be seen for the $15^{\circ}$ control target group, which shows the shortest latencies when the cue is presented at $15^{\circ}$ or $20^{\circ}$. The more diffuse cueing effect seen in the $15^{\circ}$ block may be due to cortical magnification at greater eccentricities (Rovamo and Virsu, 1979).

Next, we compared adapted saccades to control saccades. The critical aspects of these data are 1) the location of the cue that produces the shortest latencies, and 2) the overall latency curve of the adapted saccades plotted as a function of the cue location. If the cue results in more efficient visual processing of the target, then the shortest latencies should occur when the cue is flashed at the visual target location (and not at the saccade goal), i.e., $15^{\circ}$, and in addition, the latency curve should be similar to that of the $15^{\circ}$ control saccade curve. On the other hand, if the cue results in faster saccade planning, then the shortest latencies should occur when the cue is flashed at the saccade goal location (and not at the visual target location), i.e., $10^{\circ}$, and in addition the curve should match that of the $10^{\circ}$ control saccade curve. 
Adapted saccade trials-latencies

Figure $3 A$ shows a block of adapted saccade trials from a typical subject. Saccade endpoint is plotted as a function of trial number. As can be seen, saccade amplitude/endpoint decreased gradually within the first 50 trials from $15^{\circ}$ to $>10^{\circ}$ and then remained around there for the remainder of the trials. We separated the adapted saccade trials into the saccade amplitude ranges described in the Materials and Methods section. The pink shaded region depicts the adapted saccade group, i.e., saccades with endpoints within $\pm 2^{\circ}$ of the shifted target position. The gray shaded region depicts the unadapted saccade group, i.e., saccades with endpoints within $\pm 2^{\circ}$ of the initial target position.

In Figure 3B, we plot mean saccade latency of the adapted saccade group for each flashed cue position (red line) for the same subject. For comparison, we have also plotted the control latency data from Figure $2 C$ for the $10^{\circ}$ (dashed gray line) and $15^{\circ}$ control saccade (dotted gray line) conditions. The adapted saccade latencies appear to follow the latency pattern for the $10^{\circ}$ control saccade condition and not that of the $15^{\circ}$ control saccade condition. Specifically, the adapted saccade latencies are shortest when the cue is flashed at the goal of the adapted saccade $\left(10^{\circ}\right)$ and not the location of the visual target that triggered the saccadic movement $\left(15^{\circ}\right)$. In addition, the overall adapted latency curve across all cue positions appears to more closely follow the $10^{\circ}$ control saccade curve than the $15^{\circ}$ curve. If the cue most effectively facilitated processing of the visual target, we would expect that the shortest latencies would be at the $15^{\circ} \mathrm{cue}$ location and that the adapted latency curve would follow that of the $15^{\circ}$ control saccade condition.

Across all cues and all subjects, adapted saccades had an average latency of $156 \mathrm{~ms}(\mathrm{SD}=37), 10^{\circ}$ control saccades had a latency of $164 \mathrm{~ms}(40)$, and $15^{\circ}$ control saccades had a latency of $170 \mathrm{~ms}$ (41). A one-way ANOVA with an accompanying post hoc Student-Newman-Keuls test (SNK) revealed significant differences between the three latencies $\left(F_{(2,3136)}=27.248, p<0.001\right.$; SNK, $p<0.05)$.

To quantify how saccade latency was affected by the cue alone, we normalized the latencies in the cued trials relative to the nocue trials. We did this by calculating the mean latency for the no-cue trials for each subject and then subtracting this value from each of the cued trial latencies. In Figure $3 C$, we show the normalized latencies for the same subject as in Figure $3 B$. As can be seen for the control saccade data, the $10^{\circ}$ and $15^{\circ}$ curves are now slightly shifted relative to one another compared with Figure $3 B$.

Figure $3 D$ shows the normalized mean latencies across all subjects for the adapted (red line) as well as the $10^{\circ}$ and $15^{\circ}$ control saccades. Here, the adapted curve appears to follow the curve for the $10^{\circ}$ control saccades much better than it does the $15^{\circ}$ control saccade curve. In addition, the adapted saccade curve has the shortest latencies at the $10^{\circ}$ cue position, and not the $15^{\circ}$ cue condition. The normalized latencies also reveal how the cue in- fluences latency at each position relative to the no-cue condition (horizontal dotted line). For example, in the adapted curve, the shortest latencies occur when the cue is flashed at $10^{\circ}$, during which saccade latencies are on average $18 \mathrm{~ms}$ shorter than during the no-cue condition, whereas they are $\sim 21 \mathrm{~ms}$ longer when the cue is flashed on the opposite side of fixation $\left(-5^{\circ}\right)$.

We performed univariate ANOVAs on the normalized saccade latencies for each group separately with cue position as a factor finding a significant effect of cue position on each group $\left(10^{\circ}=F_{(6,1065)}=8.993, p<0.001,15^{\circ}=F_{(6,854)}=11.57, p<\right.$ 0.001 , adapted $\left.=F_{(6,763)}=16.21, p<0.001\right)$. Separate post hoc analyses (SNK) revealed that for both the $10^{\circ}$ control saccade group as well as the adapted group, latencies for the $10^{\circ}$ cue location were significantly different from all other cue locations $(p<0.05)$. In the $15^{\circ}$ control saccade group, the latencies for the $15^{\circ}$ cue location were significantly different from all other cue locations except $20^{\circ}(p<0.05)$.

We compared the overall cueing-effect curves to determine which control saccade curve $\left(10^{\circ}\right.$ or $\left.15^{\circ}\right)$ the adapted curve matched the closest. For each individual subject as well as across all subjects, we calculated the magnitude of the difference between the mean adapted saccade latency and the means of each of the two control saccade latencies at each cue location and then averaged these magnitudes across the cue locations. If the cue speeds up processing of the visual target, then the difference magnitude should be smaller between the adapted curve and the $15^{\circ}$ control saccade curve. On the other hand, if the cue speeds up 


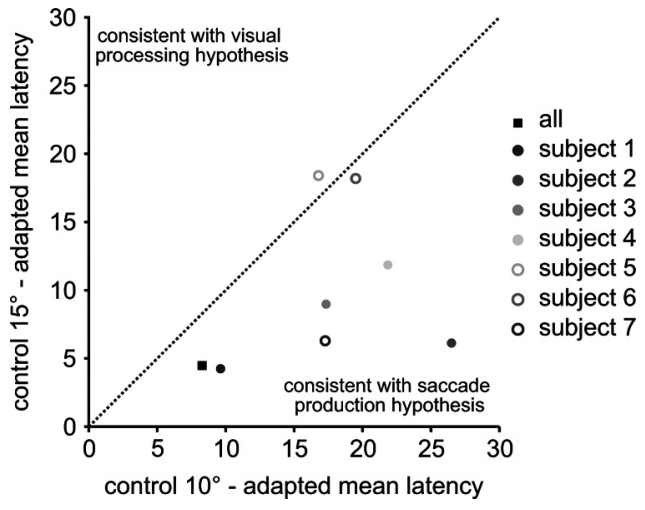

Figure 4. Direct comparison between motor and visual hypotheses. The $x$-axis plots the mean absolute difference between the average adapted saccade latencies and the average $10^{\circ}$ control latencies. The differences between the mean latencies at each cue location were calculated and then all 7 differences were averaged. The $y$-axis plots the mean absolute difference between the average adapted saccade latencies and the average $15^{\circ}$ control latencies. Each dot represents one subject and the black filled square represents the mean difference from all subjects. The diagonal line represents unity. A data point below the line is consistent with the motor production hypothesis because the difference between the adapted saccade and the $10^{\circ}$ control target latencies are smaller than the difference between the adapted saccade the $15^{\circ}$ control target latencies. A data point above the line of unity is consisted with the visual processing hypothesis.

Table 1. Correlations

\begin{tabular}{lll}
\hline Subject & Adapted $/ 15^{\circ}$ control (7) & Adapted $/ 10^{\circ}$ control (7) \\
\hline All & $r=0.469$ & $r=0.918^{* *}$ \\
Subject 1 & -0.347 & $0.902^{* *}$ \\
Subject 2 & 0.071 & $0.890^{* *}$ \\
Subject 3 & 0.055 & 0.614 \\
Subject 4 & -0.074 & 0.444 \\
Subject 5 & 0.554 & 0.256 \\
Subject 6 & 0.365 & 0.595 \\
Subject 7 & $0.966^{* *}$ & $0.926^{* *}$ \\
\hline
\end{tabular}

${ }^{* *} p<0.01$.

production of the saccade, then the difference magnitude should be smaller between the adapted saccade curve and the $10^{\circ}$ control saccade curve. In Figure 4, the diagonal dotted line is the line of unity. Data points falling below this line are consistent with the saccade production hypothesis whereas data points falling above the line are consistent with the visual processing hypothesis. The data points for all subjects except one fell below the line of unity, consistent with the saccade production hypothesis.

We also performed a correlational analysis between the mean adapted latencies and the control saccade latencies ( 7 mean latencies for each of the 7 cue locations). A higher correlation between one control saccade group $\left(15^{\circ}\right.$ control vs $10^{\circ}$ control) and the adapted saccade data compared with the other control saccade group would demonstrate that the curves match better. As can be seen in Table 1, saccade latency correlations were consistently higher between the adapted saccade and the $10^{\circ}$ control conditions (corresponding to the saccade production hypothesis) than between the adapted saccade and the $15^{\circ}$ control condition (corresponding to the visual processing hypothesis) in all but one subject (subject 7). This subject nevertheless had a high correlation between the $10^{\circ}$ control and adapted saccade latencies, but the overall pattern of latencies for this subject was similar across all three conditions, making it difficult to dissociate between the visual processing and saccade production hypotheses.

Together, the results suggest that the cue that has its greatest influence when flashed at the upcoming saccade goal location and not at the visual target location.

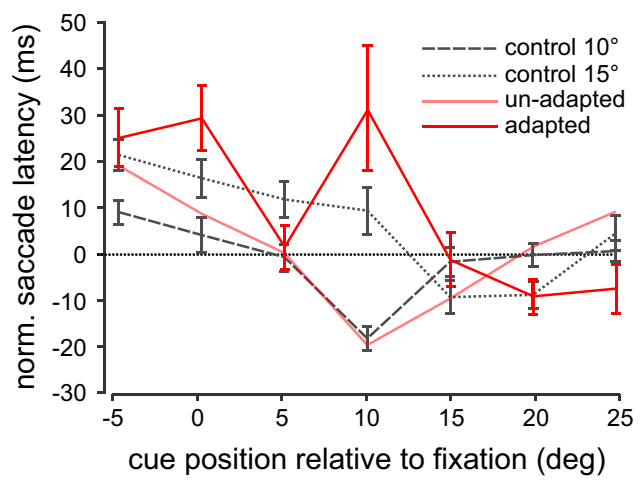

Figure 5. Unadapted saccade latencies. Average unadapted normalized saccade latencies across all subjects for the $15^{\circ}$ to $10^{\circ}$ adapted saccade condition plotted as a function of cue position (thicker red line) as in Figure 3. Unadapted saccades were defined as saccades that fell within $\pm 2^{\circ}$ of the first target position as shown by the gray region in Figure $3 A$. For comparison, the $10^{\circ}$ and $15^{\circ}$ control and adapted saccade latencies are replotted as well as the adapted saccade latency curve from Figure $4 E$ (light red line).

\section{Unadapted saccades-latencies}

One test of the above conclusion is that the influence of the cue should depend on saccade endpoint, e.g., if the endpoint is $15^{\circ}$ to the right, then a cue flashed at that location should have the greatest influence, regardless of the visual target location. To test this, we examined the group of unadapted saccades that occurred during the adapted saccade trials. These were saccades in the adaptation block which had endpoints within $\pm 2^{\circ}$ of the initial target location. Across all subjects, there were 333 unadapted saccades in total, $31 \%$ of which occurred during the second half of the adaptation block (see supplemental Fig. 1, available at www.jneurosci.org as supplemental material, for endpoint data for a second subject). Across all cues, unadapted saccades had an average latency of $162 \mathrm{~ms}(\mathrm{SD}=42)$. A one-way ANOVA comparing these saccades and the $15^{\circ}$ and $10^{\circ}$ control saccades was significant $\left(F_{(2,2589)}=7.497, p<0.01\right)$. A post hoc $\mathrm{SNK}$ revealed that the latencies of the unadapted saccades were not different from the $10^{\circ}$ control saccade latencies $(p>0.05)$.

In the same manner as for Figure $3 D$, we normalized the latencies for the unadapted saccades. Figure 5 shows the mean normalized latency curves for the unadapted saccades occurring during the adapting paradigm across all subjects (thick red line). The lighter trace of the same color in this figure shows the data from the adapted saccade group for comparison. In addition, the $15^{\circ}$ and $10^{\circ}$ control saccade curves are also plotted. The figure shows that the unadapted curve (thick red line) does not follow the same pattern of latencies across cue positions as the adapted curve (light red line). Rather, the curve appears to fit better with the $15^{\circ}$ saccade curve. This was quantified using correlation analysis as performed above; the correlation between the unadapted latencies and the $15^{\circ}$ control latencies was higher at 0.735 ( $p=0.06, n=7$ ) compared with the correlation between the unadapted latencies and the $10^{\circ}$ control latencies at -0.172 $(p=0.712, n=7)$. Thus, for both unadapted and adapted saccades, the cue has the greatest influence on saccade latencies when it appears at the saccade endpoint.

To ensure that these findings are unrelated to the process of saccade adaptation itself, we performed an additional analysis where we separated adaptation trials into 3 epochs- early, middle, and late adaptation. We found the same effect of cue on adaptation latencies across all three epochs (see supplemental Fig. 2, available at www.jneurosci.org as supplemental material). 


\section{Saccade amplitude and dynamics}

Since adaptation is often incomplete, adapted saccades toward lower gains tend to have larger amplitudes than normal saccades for the same final target location (Desmurget et al., 1998; Hopp and Fuchs, 2004; Cotti et al., 2007). In addition, when matched for amplitude, adapted saccades also have longer durations and decreased peak velocities compared with normal saccades (Frens and van Opstal, 1994; Collins et al., 2008). We investigated whether these differences also occurred in our data, and whether the cue at either the saccade goal or visual target had an additional influence on amplitude and dynamics, such as peak velocity and duration, for the adapted saccades.

We found that average saccade amplitude in the adapted trials was reduced by $80.4 \%$ (from $14.61^{\circ}$ [ $15^{\circ}$ control amplitude] to $10.6^{\circ}$ [adapted amplitude] $=4.01^{\circ}$ ) of the required displacement $\left(15^{\circ}\right.$ to $\left.10^{\circ}=5^{\circ}\right)$ across all cue conditions combined. This is consistent with previous studies (Desmurget et al., 1998; Hopp and Fuchs, 2004; Cotti et al., 2007). However, we found that within the adapted saccade group, saccade amplitudes were significantly smaller when the cue was flashed at the saccade goal $\left(F_{(7,870)}=12.662, p<0.001, \mathrm{SNK}, p<0.05\right)$ but were not significantly larger when the cue was flashed at the visual target location $(p>0.05)$. Within the $10^{\circ}$ control saccades, amplitudes were not smaller for the $5^{\circ}$ compared with the $15^{\circ}$ cue location $(\mathrm{SNK}>0.05)$.

To compare saccade dynamics, we equalized amplitudes at each cue location between the adapted and the $10^{\circ}$ control saccade groups (see Materials and Methods). We compared average velocity profiles between the adapted (red) and the $10^{\circ}$ control saccades (gray) when the cue was flashed at the $15^{\circ}$-visual target (Fig. $6 \mathrm{~A}$ ) and at the $10^{\circ}$-saccade goal (Fig. $6 \mathrm{~B}$ ) locations. In both figures, the average \pm the SEM of all velocity profiles aligned at saccade onset is plotted.

Figure $6 \mathrm{~A}$ shows that within these trials with equalized amplitudes $(p>0.05)$, when the cue was flashed at the visual target location, peak velocity was decreased for the adapted saccades (red traces) compared with the $10^{\circ}$ control group $\left(F_{(1,128)}=\right.$ $21.392, p<0.01)$. Figure $6 B$ shows that when the cue was flashed at the upcoming saccade goal location, in contrast to the cue being flashed at the visual target location, peak velocity was not significantly different from the control data $(p>0.05)$.

Figure $6 C$ plots peak velocity as a function of cue position for the adapted (red line) and control (gray dashed line) saccades with equivalent amplitudes. Overall peak velocity was significantly different for the adapted saccades compared with the control saccades $\left(F_{(1,1132)}=11.731, p<0.05\right)$. Within the adapted saccades, we compared peak velocity across cue conditions to the no-cue condition and found that the $10^{\circ}$ and the $25^{\circ}$ cue location was significantly different from the no-cue condition $\left(F_{(7,549)}=\right.$ $3.64, p<0.001$, SNK $<0.05)$. The $10^{\circ}$ cue condition was not different from the no-cue condition in the control group $(p<$ $0.05)$. Figure $6 D$ plots the duration of the deceleration period as a function of cue position. Overall the duration of the deceleration period was higher for the adapted saccades compared with the control saccades $\left(F_{(1,1132)}=28.455, p<0.001\right)$. Within the adapted trials, the $10^{\circ}$ cue location had the lowest duration but was only significantly different from the $25^{\circ}$ cue location $\left(F_{(7,549)}=\right.$ $2.983, p<0.01, \mathrm{SNK}<0.05)$. There were no significant differences for across any cue condition within the control trials $(p>0.05)$. The duration of the acceleration period was significantly higher for the control trials $(36.3 \mathrm{~ms})$ than for the adapted trials $(35.5$ $\left.\mathrm{ms} ; F_{(1,1132)}=27.969, p<0.05\right)$ but there were no within-group cue differences $(p>0.05)$.
To summarize, we replicated previous findings by showing that overall, adapted saccades differ from normal saccades of equivalent amplitude, with reduced peak velocities and longer acceleration and deceleration period durations. However, when the cue was presented at the saccade goal location, peak velocity and deceleration duration did not follow this pattern.

\section{Discussion}

We investigated the mechanism underlying saccadic facilitation, the well known decrease in saccade latency following a behaviorally irrelevant exogenous cue. We asked whether the cue influenced processing of the visual target or planning of the saccade by using saccadic adaptation to dissociate the locations of the visual target and the saccade goal. We found that adapted saccades were most strongly influenced when the cue was flashed at the upcoming saccade goal location and not when the cue was flashed at the visual target location. Therefore, we conclude that the attentional cue had a predominant influence on the late motor programming of the saccade rather than on the early visual processing of the target.

An attentional cue presented at the saccade goal had three main effects on adapted saccades: first, latencies were shorter compared with when the cue was presented elsewhere; second, saccade amplitude was smaller; and finally, the velocity profile was similar to that of control saccades rather than the velocity profile of a typical adapted saccade.

\section{Latency, velocity, and amplitude effects}

For control saccades, we found that saccades had shorter latencies when a cue was presented at the same location as the target and longer latencies when the cue-target distance was greater, compared with the condition with no cue present. This is in agreement with earlier studies in which the visual target and saccade goal locations were undifferentiated (Shepherd et al., 1986; Crawford and Muller, 1992; Bell et al., 2004; Fecteau et al., 2004). Critically, when we separated the visual target and saccade goal locations by adapting saccades to a target at $15^{\circ}$ eccentricity down to $\sim 10^{\circ}$ in amplitude, the pattern of cueing effects for the adapted saccades was similar to that which was observed for control saccades to a target at an eccentricity of $10^{\circ}$. In both cases, saccade latencies were shortest when the cue was flashed at $10^{\circ}$ and gradually increased for more distant cues. In the $10^{\circ}$ control saccade group, the optimal cue location corresponded with both the saccade goal and the target location. However, in the adapted saccade group, this corresponded with the saccade goal location and not the visual target location.

Even within the adaptation block, saccades with endpoints that were closer to $15^{\circ}$ showed a pattern of latencies consistent with the $15^{\circ}$ control latencies. These results also exclude the possibility that our results arose from an overall difference in performance between the adaptation and control blocks, e.g., an adaptation task set. It could be argued that the saccades with larger amplitudes took place within the first few trials before adaptation occurred. However, $\sim 1 / 3$ of these saccades with greater amplitudes occurred within the second half of the adaptation block (see supplemental Fig. 1, available at www.jneurosci. org as supplemental material). Therefore, our results support the hypothesis that the cue had the greatest influence when it was presented at the saccade goal rather at the visual target.

Previous studies have shown that adapted saccades have different dynamics from those of normal saccades (Frens and van Opstal, 1994; Straube and Deubel, 1995; Collins et al., 2008). We reproduced the findings of Collins et al. (2008), who showed that adapted saccades have a longer deceleration period than control 
A
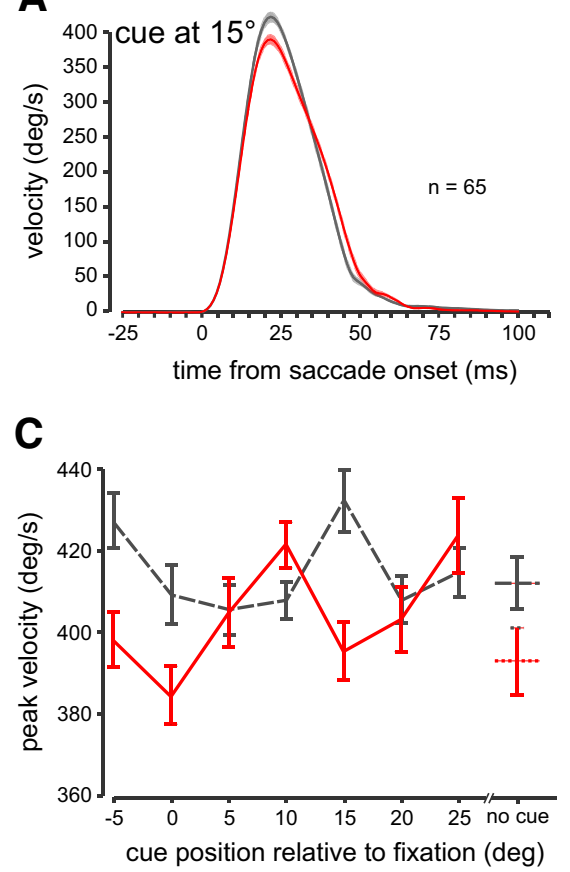

B

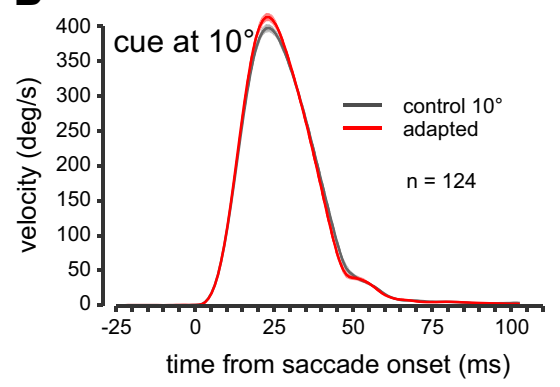

D

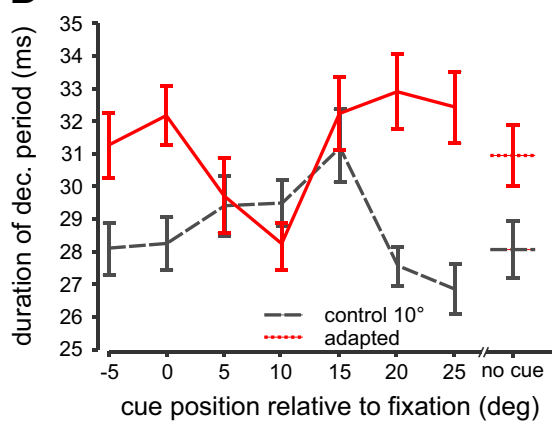

latencies at that cue location. Finally, it has been shown that saccade adaptation to targets presented at one amplitude partially transfers to targets at other nearby amplitudes (Noto et al., 1999; Hopp and Fuchs, 2004). Specifically, Noto et al. (1999) showed (in monkeys) that adaptation to a $15^{\circ}$ target, transfers $\sim 60-70 \%$ to a $10^{\circ}$ target. Taking this into account, if subjects were indeed making a saccade to the $10^{\circ}$ cue, they should have shown some adaptation transfer and accordingly made saccades of $\sim 8.5^{\circ}$. Instead, their saccades were $9.9^{\circ}$ on average when the cue was flashed at the $10^{\circ}$, comparable to saccade amplitudes for the $10^{\circ}$ control block.

\section{Possible neural mechanisms}

We believe that our results imply a spatial interaction between the visual location of the attentional cue and the saccade goal location. One area where this could occur is the superior colliculus (SC). The SC contains topographic visual and motor maps (Scudder et al., 2002), and receives inputs from brain regions such as the prefrontal and parietal cortices (Lui et al., 1995; Clower et al., 2001; May, 2006). Recent studies have shown neuronal correlates of attention capture in the SC (Bell et

saccades with the same amplitude. However, in our task, when the cue was flashed at the saccade goal location, the velocity profiles of adapted saccades became more similar to those of the control saccades. We propose therefore that the cue provided a visual signal that interacted with the saccade goal, i.e., an averaging effect (Edelman and $\mathrm{Xu}, 2009$ ) resulting in a saccade that had similar dynamics to the nonadapted saccades. This is consistent with the finding that visually guided saccades have higher velocities than non-visually guided saccades (Smit et al., 1987; Smit and Van Gisbergen, 1989; Gnadt et al., 1991; Van Gelder et al., 1997; Amador et al., 1998; Ohno et al., 2000; Edelman and Goldberg, 2001; Edelman et al., 2006) and could account for the fact that when the cue is presented at the saccade goal location, the amplitudes of adapted saccades are more similar to the controls.

As with any study of exogenous saccade cueing, it could be that both the adapted and control saccade curves show a reduction in saccade latencies when the cue location coincides with the saccade goal location simply because subjects are essentially making a premature saccade to the cue rather than the target. However, the data argue against this possibility. First, the latency reductions are at most $15 \mathrm{~ms}$ compared with the overall latencies across the rest of the cue locations, whereas the timing difference between the cue and the target was $50 \mathrm{~ms}$. However, this smaller latency effect could be explained if some saccades were made to the cue and others to the target. Therefore, we examined the latency distributions when the cue was presented at the saccade goal, but did not find any bimodal distributions that would be suggestive of this possibility. Instead, all latency distributions showed a single peak with equivalent widths. In addition, during adaptation, the saccade is triggered by the target which is at $15^{\circ}$ location. We would expect that if subjects were making premature saccades to the cue, they would be more likely to do so when the cue was flashed at $15^{\circ}$ (close to the target) resulting in shortest al., 2004; Fecteau et al., 2004). For example, Fecteau et al. (2004) found stronger target-related activity when a cue and target appeared in the same location, because the target-related activity built on the previous cue-related activity. This activity was significantly correlated with saccadic reaction time; stronger target-related activity led to shorter reaction times. Differences in activity at the same location in the SC have been shown to lead to changes in saccade latency, amplitude and velocity profiles (Stanford et al., 1996), which may explain our findings. With respect to our task, we hypothesize that it is the saccade goal location rather than the visual target location that interacts or averages with the cue, suggesting that information about the adapted saccade goal is represented in the SC; with the activity related to the saccade building on the previous cue-related activity resulting in a shorter saccade latency. This is partially supported by physiological evidence, however controversy remains as to whether the SC encodes the saccade goal location as opposed to the target location for adapted saccades (Fitzgibbon et al., 1986; Melis and van Gisbergen, 1996; Frens and Van Opstal, 1997; Edelman and Goldberg, 2002; Takeichi et al., 2005, 2007; Edelman and Xu, 2009).

\section{Effects of attention on visual and motor processing}

Many previous studies have demonstrated tasks and situations where attentional cueing clearly facilitates early sensory processing stages (Jonides, 1981; Egeth and Yantis, 1997; Snowden, 2002). Our findings do not dispute these studies, rather, they reveal the mechanisms involved in classic attentional cueing tasks with saccades, which have been widely used for many decades (Posner et al., 1982; Abrams and Dobkin, 1994). Within this context, our findings expand on those of Fecteau and Munoz (2005), who show evidence for attentional mechanisms occurring at later motor stages of processing in the SC, by showing that attention influences processing of the saccade goal rather than the visual target. 


\section{References}

Abrams RA, Dobkin RS (1994) Inhibition of return: Effects of attentional cueing on eye movement latencies. J Exp Psychol Hum Percept Perform 20:467-477.

Albano JE, King WM (1989) Rapid adaptation of saccadic amplitude in humans and monkeys. Invest Ophthalmol Vis Sci 30:1883-1893.

Amador N, Schlag-Rey M, Schlag J (1998) Primate antisaccades. I. Behavioral characteristics. J Neurophysiol 80:1775-1786.

Bahcall DO, Kowler E (1999) Illusory shifts in visual direction accompany adaptation of saccadic eye movements. Nature 400:864-866.

Bahcall DO, Kowler E (2000) The control of saccadic adaptation: implications for the scanning of natural scenes. Vision Res 40:2779-2796.

Bell AH, Fecteau JH, Munoz DP (2004) Using auditory and visual stimuli to investigate the behavioral and neuronal consequences of reflexive covert orienting. J Neurophysiol 91:2172-2184.

Brainard DH (1997) The Psychophysics Toolbox. Spat Vis 10:433-436.

Bridgeman B, Hendry D, Stark L (1975) Failure to detect displacement of the visual world during saccadic eye movements. Vision Res 15:719-722.

Clower DM, West RA, Lynch JC, Strick PL (2001) The inferior parietal lobule is the target of output from the superior colliculus, hippocampus, and cerebellum. J Neurosci 21:6283-6291.

Collins T, Semroud A, Orriols E, Doré-Mazars K (2008) Saccade dynamics before, during, and after saccadic adaptation in humans. Invest Ophthalmol Vis Sci 49:604-612.

Cotti J, Guillaume A, Alahyane N, Pelisson D, Vercher JL (2007) Adaptation of voluntary saccades, but not of reactive saccades, transfers to hand pointing movements. J Neurophysiol 98:602-612.

Crawford TJ, Muller HJ (1992) Spatial and temporal effects of spatial attention on human saccadic eye movements. Vision Res 32:293-304.

Desmurget M, Pélisson D, Urquizar C, Prablanc C, Alexander GE, Grafton ST (1998) Functional anatomy of saccadic adaptation in humans. Nat Neurosci 1:524-528.

Deubel H (1991) Adaptive control of saccade metrics. In: Presbyopia research (Obrecht G, Stark L, eds), pp 93-100. New York: Plenum.

Deubel H, Wolf W, Hauske G (1986) Adaptive gain control of saccadic eye movements. Hum Neurobiol 5:245-253.

Edelman JA, Goldberg ME (2001) Dependence of saccade-related activity in the primate superior colliculus on visual target presence. J Neurophysiol 86:676-691.

Edelman JA, Goldberg ME (2002) Effect of short-term saccadic adaptation on saccades evoked by electrical stimulation in the primate superior colliculus. J Neurophysiol 87:1915-1923.

Edelman JA, Xu KZ (2009) Inhibition of voluntary saccadic eye movement commands by abrupt visual onsets. J Neurophysiol 101:1222-1234.

Edelman JA, Valenzuela N, Barton JJ (2006) Antisaccade velocity, but not latency, results from a lack of saccade visual guidance. Vision Res 46:1411-1421.

Egeth HE, Yantis S (1997) Visual attention: control, representation, and time course. Annu Rev Psychol 48:269-297.

Erkelens CJ, Hulleman J (1993) Selective adaptation of internally triggered saccades made to visual targets. Exp Brain Res 93:157-164.

Fecteau JH, Munoz DP (2005) Correlates of capture of attention and inhibition of return across stages of visual processing. J Cogn Neurosci 17:1714-1727.

Fecteau JH, Bell AH, Munoz DP (2004) Neural correlates of automatic and goal-driven biases in orienting spatial attention. J Neurophysiol 92:17281737.

Fitzgibbon EJ, Goldberg ME, Segraves MA (1986) Short term saccadic adaptation in the monkey. In: Adaptive processes in visual and oculomotor systems (Keller BL, Zee DS, eds), pp 329-333. Oxford: Pergamon.

Frens MA, van Opstal AJ (1994) Transfer of short-term adaptation in human saccadic eye movements. Exp Brain Res 100:293-306.

Frens MA, Van Opstal AJ (1997) Monkey superior colliculus activity during short-term saccadic adaptation. Brain Res Bull 43:473-483.

Fuchs AF, Reiner D, Pong M (1996) Transfer of gain changes from targeting to other types of saccade in the monkey: constraints on possible sites of saccadic gain adaptation. J Neurophysiol 76:2522-2535.

Gnadt JW, Bracewell RM, Andersen RA (1991) Sensorimotor transformation during eye movements to remembered visual targets. Vision Res 31:693-715.

Hopp JJ, Fuchs AF (2004) The characteristics and neuronal substrate of saccadic eye movement plasticity. Prog Neurobiol 72:27-53.

Jonides J (1981) Voluntary versus automatic control over the mind's eye's movement. In: Attention and performance IX (Long JB, Baddeley AD eds), pp 187-203. Hillsdale, NJ: Erlbaum.

Lui F, Gregory KM, Blanks RH, Giolli RA (1995) Projections from visual areas of the cerebral cortex to pretectal nuclear complex, terminal accessory optic nuclei, and superior colliculus in macaque monkey. J Comp Neurol 363:439-460.

May PJ (2006) The mammalian superior colliculus: laminar structure and connections. Prog Brain Res 151:321-378.

McLaughlin SC (1967) Parametric adjustment in saccadic eye movements. Percept Psychophys 2:359-362.

Melis BJ, van Gisbergen JA (1996) Short-term adaptation of electrically induced saccades in monkey superior colliculus. J Neurophysiol 76:17441758 .

Noto CT, Robinson F (2001) Visual error is the stimulus for saccadic gain adaptation. Cogn Brain Res 12:301-305.

Noto CT, Watanabe S, Fuchs AF (1999) Characteristics of simian adaptation fields produced by behavioral changes in saccade size and direction. J Neurophysiol 81:2798-2813.

Ohno K, Matsuzaki H, Yamada T, Yoshida H, Shimizu K (2000) The difference in saccadic parameters among several visually guided tasks. Jpn Ophthalmol 44:695.

Panouillères M, Weiss T, Urquizar C, Salemme R, Munoz DP, Pélisson D (2009) Behavioral evidence of separate adaptation mechanisms controlling saccade amplitude lengthening and shortening. J Neurophysiol 101:1550-1559.

Pelli DG (1997) The VideoToolbox software for visual psychophysics: transforming numbers into movies. Spat Vis 10:437-442.

Posner MI, Cohen Y (1984) Components of visual attention. In: Attention and performance X (Bouma H, Bouwhuis DG, eds), pp 531-556. Hillsdale, NJ: Erlbaum.

Posner MI, Cohen Y, Rafal RD (1982) Neural systems control of spatial orienting. Philos Trans R Soc Lond B Biol Sci 298:187-198.

Rovamo J, Virsu V (1979) An estimation and application of the human cortical magnification factor. Exp Brain Res 37:495-510.

Scudder CA, Kaneko CS, Fuchs AF (2002) The brainstem burst generator for saccadic eye movements: a modern synthesis. Exp Brain Res 142 439-462.

Semmlow JL, Gauthier GM, Vercher JL (1989) Mechanisms of short-term saccadic adaptation. J Exp Psychol Hum Percept Perform 15:249-258.

Shepherd M, Findlay JM, Hockey RJ (1986) The relationship between eye movements and spatial attention. Q J Exp Psychol A 38:475-491.

Smit AC, Van Gisbergen JA (1989) A short-latency transition in saccade dynamics during square-wave tracking and its significance for the differentiation of visually-guided and predictive saccades. Exp Brain Res 76:64-74.

Smit AC, Van Gisbergen JA, Cools AR (1987) A parametric analysis of human saccades in different experimental paradigms. Vision Res 27:17451762.

Snowden RJ (2002) Visual attention to color: parvocellular guidance of attentional resources? Psychol Sci 13:180-184.

Stanford TR, Freedman EG, Sparks DL (1996) Site and parameters of microstimulation: evidence for independent effects on the properties of saccades evoked from the primate superior colliculus. J Neurophysiol 76: 3360-3381.

Straube A, Deubel H (1995) Rapid gain adaptation affects the dynamics of saccadic eye movements in humans. Vision Res 35:3451-3458.

Straube A, Fuchs AF, Usher S, Robinson FR (1997) Characteristics of saccadic gain adaptation in rhesus macaques. J Neurophysiol 77:874-895.

Takeichi N, Kaneko CR, Fuchs AF (2005) Discharge of monkey nucleus reticularis tegmenti pontis neurons changes during saccade adaptation. J Neurophysiol 94:1938-1951.

Takeichi N, Kaneko CR, Fuchs AF (2007) Activity changes in monkey superior colliculus during saccade adaptation. J Neurophysiol 97:4096-4107.

Van Gelder P, Lebedev S, Tsui WH (1997) Peak velocities of visually and nonvisually guided saccades in smooth-pursuit and saccadic tasks. Exp Brain Res 116:201-215.

Walker R, Kentridge RW, Findlay JM (1995) Independent contributions of the orienting of attention, fixation offset and bilateral stimulation on human saccade latencies. Exp Brain Res 103:294-310.

Wallman J, Fuchs AF (1998) Saccadic gain modification: visual error drives motor adaptation. J Neurophysiol 80:2405-2416.

Wenban-Smith MG, Findlay JM (1991) Express saccades: is there a separate population in humans? Exp Brain Res 87:218-222. 